\title{
Zur Langzeitökologie des Lärchen-Arvengürtels in den südlichen Walliser Alpen
}

\section{Petra Kaltenrieder, Willy Tinner und Brigitta Ammann}

Institut für Pflanzenwissenschaften der Universität Bern, Abteilung Paläoökologie, Altenbergrain 21, CH-3013 Bern; e-mail: petra.kaltenrieder@ips.unibe.ch

Manuskript angenommen am 24. Juli 2005

\begin{abstract}
Kaltenrieder P., Tinner W. and Ammann B. 2005. Long-term vegetation history at timberline in the Swiss Alps (Alpe d'Essertse, VS). Bot. Helv. 115: 137-154.

Palaeoecological studies in the "Alpe d'Essertse" area have provided much information about vegetation changes and timberline fluctuations during the Holocene. In this study we repeated previous biostratigraphic investigations using plant macrofossils to improve their temporal and taxonomic resolution and to test their reliability. By analyzing $0.5-\mathrm{cm}$ layers of a lake sediment we reached a temporal resolution of 44 years, and we were able to reconstruct vegetation changes in the surrounding area at species level. The sedimentary record analyzed extends from the Late-Glacial to the late Holocene. Alpine grasslands (12'000-11'000 cal. BP) were afforested by Larix decidua, Juniperus nana, and Pinus cembra (11,000-9'600 cal. B.P.). Stable subalpine larch-stone pine-forests (9'600-4'900 cal. BP) were followed by shrublands and meadows as a consequence of the climatically and anthropogenically induced destruction of forest vegetation (4'900-2'600 cal. BP). Changes in the abundance of $P$. cembra and $L$. decidua needles as well as changes of the other taxa were consistent with those found in previous studies from the same lake. Our results demonstrate that plant-macrofossil records can be reproduced spatially and temporally on separate cores with independent ${ }^{14} \mathrm{C}$ chronologies.
\end{abstract}

Key words: Plant macrofossils, timberline, vegetation history, palaeoecology, climate change, human impact.

\section{Einleitung}

Aus ökologischer Sicht wird die Waldgrenze in erster Linie durch einen Wärmemangel während der Vegetationszeit verursacht (Tranquillini 1979; Kupfer und Cairns 1996). Da Klimaänderungen die Höhenlage der Waldgrenze beeinflussen, wurde die Lage der alpinen Waldgrenze seit der letzten Eiszeit an vielen Lokalitäten untersucht, um die Klimageschichte zu rekonstruieren (Bortenschlager 1972; Zoller 1977; Reasoner und Hickman 1989; Wegmüller und Lotter 1990; Burga 1991; Carrara 
et al. 1991; Kullman 1995; Wick und Tinner 1997; Haas et al. 1998; Barnekow und Sandgren 2001; Reasoner et al. 2001). Hingegen haben sich nur wenige Untersuchungen den Veränderungen der Vegetationszusammensetzung an der Waldgrenze und den dafür verantwortlichen Umweltfaktoren gewidmet. Wichtige Erkenntnisse hierüber wurden auf der Alpe d'Essertse im südlichen Wallis gewonnen (Tinner et al. 1996, Wick und Tinner 1997). Pollenanalysen und Makrorestanalysen zeigten auf, dass die Waldgrenze nach der letzten Eiszeit zuerst angestiegen ist, dann aufgrund von kürzeren Kaltphasen fluktuiert hat, und schliesslich unter klimatischem und menschlichem Einfluss wieder gesunken ist (Tinner et al. 1996, Wick und Tinner 1997).

Die Aussagekraft der bisherigen Untersuchungen auf der Alpe d'Essertse war aber aus methodischen Gründen begrenzt. Die Pollenanalysen liessen für viele Taxa keine Aussagen zum Vorkommen einzelner Arten zu. Diese Begrenzung der taxonomischen Auflösung betrifft insbesondere die im Bereich der Waldgrenze sehr wichtige Familie der Ericaceen. Bei den Makrorestanalysen betrug die zeitliche Auflösung im Mittel 162 Jahre, ein für derartige Untersuchungen typischer Wert, der aber bei raschen Vegetationsveränderungen ungenügend sein kann (Tinner und Ammann 2001). Ferner herrscht unter Fachleuten eine allgemeine Skepsis, ob eine makrorestanalytische Studie in einem Gebiet reproduzierbar ist, da die Makroreste aufgrund ihrer Grösse nicht einheitlich im Raum verteilt werden (Birks und Birks 1980). Aufgrund der aufwändigen und zeitraubenden Analysen wurde bisher nur vereinzelt mehr als eine makrorestanalytische Studie im gleichen Sedimentbecken durchgeführt (Schneider und Tobolski 1985). Diese zeigten, dass die biostratigraphischen Muster im Allgemeinen reproduzierbar sind; Die Auflösung und die chronologische Genauigkeit waren aber ungenügend, um allgemeingültige Aussagen über die räumlich-zeitliche Reproduzierbarkeit makrorestanalytischer Untersuchungen zu machen.

Das Hauptziel der vorliegenden Arbeit bestand deshalb darin, herauszufinden, ob die Resultate der früheren pollen- und makrorestanalytischen Untersuchungen (Tinner et al. 1996; Wick und Tinner 1997) durch eine weitere makrorestanalytische Untersuchung reproduzierbar sind, und inwiefern durch eine höhere taxonomische und zeitliche Auflösung zusätzliche Erkenntnisse gewonnen werden können.

\section{Material und Methoden}

\section{Untersuchungsgebiet}

Die grosse Ebene der Alpe d'Essertse, Grand Tsa, (Gemeinde Hérémence) liegt im Val d'Hérémence auf 2'000 bis 2'600 m ü.M. (Tinner 1994; Tinner et al. 1996; Wick und Tinner 1997; Tinner und Theurillat 2003). Es ist eine typische Moränenlandschaft mit langgezogenen Moränenwällen, reich an Mooren und kleinen Seen oder Tümpeln (Gouillés). Das Moränenmaterial besteht hauptsächlich aus Hellglimmerschiefer (Tinner 1993). Tektonisch gehört die Alpe d'Essertse zum Kristallin der Bernharddecke. Am nördlichen Rand der Alp zieht sich ein Band von mesozoischen Sedimenten durch das Gebiet (Vallet 1950).

Die in dieser Arbeit untersuchten Sedimentkerne entstammen dem Seelein Gouillé Rion (0.5 ha, 4609'25'N, 07²1'45'E, 2'343 m ü.M.). Das in einer Karnische eingebettete Seelein ist rundum von Moränen-Hügeln umgeben, welche gegen Norden/Nordosten (N/NE) etwas steiler und höher sind als gegen Süden/Südwesten (S/SW). Um 
und in diesem Seelein wurden 1991-1996 insgesamt fünf Sedimentkerne entnommen, von denen zwei (GR-1/GR-2) untersucht wurden.

Mit einer mittleren Niederschlagsmenge von 500-700 $\mathrm{mm}$ in tieferen Lagen und 900-1'100 mm auf 2'300 m ü.M. weist das Untersuchungsgebiet ein inneralpines Trockenklima auf. Auf der Alpe d'Essertse beträgt die Jahresmitteltemperatur und die Julimitteltemperatur ungefähr $1^{\circ} \mathrm{C}$ bzw. $9^{\circ} \mathrm{C}$.

Die aktuelle Waldgrenze im Val d'Hérémence liegt auf 2'000 bis 2'250 m ü.M. (Landeskarten 1:25'000 Blatt 1306 und 1326). Bis etwa 2'000 m (je nach Exposition) herrscht ein subalpiner Fichtenwald vor (Vaccinium-Piceion). Von 2'000 bis 2'100 m wächst ein fast reiner Lärchenwald mit Rhododendron ferrugineum, Vaccinium myrtillus, $V$. vitis-idaea, $V$. gaultherioides und Homogyne alpina im Unterwuchs. Darüber findet man einzelne schön gewachsene Arven (Pinus cembra), und an feuchteren Hängen einen schmalen, aber dichten Grünerlengürtel (Alnus viridis) mit vereinzelten Vogelbeerbäumen (Sorbus aucuparia). Die potentielle, natürliche Waldgrenze kann anhand von einzelnen, gut entwickelten Arven (>5 m) auf 2'320 m, die Baumgrenze anhand von kleineren Exemplaren von bis 2 m Wuchshöhe auf 2’350-2’400 m ü.M. geschätzt werden.

Heute wird die Alpe d'Essertse während des Sommers von Eringer Kühen, weiter oben auch von Schafen und Ziegen beweidet. Der vorherrschende Vegetationstyp ist ein typischer Borstgras-Rasen (Geo-montani-Nardetum), welcher mit verschiedenen subalpinen Zwergstrauchheiden durchmischt ist. An sonnenexponierten Steilhängen wachsen Buntschwingel-Rasen (Festucetum variae), und auf windexponierten, trockenen Moränenrücken Nacktried-Rasen (Elynetum). Auf den mesozoischen Sedimenten kommt lokal auch kalkzeigende Vegetation vor. In einer etwas feuchteren Senke fanden sich gut gedeihende Spaliersträucher von Salix reticulata.

\section{Makrorestanalyse}

In der Mitte des Seeleins Gouillé Rion, in einer Wassertiefe von $760 \mathrm{~cm}$ wurden mit einem modifizierten Livingstone-Stechrohr-Bohrer (Merkt und Streif 1970) zwei parallel gelegene Sedimentkerne (GR-6 und -7) im Abstand von $1 \mathrm{~m}$ zueinander und im Abstand von $10 \mathrm{~m}$ zum früher untersuchten Sedimentkern GR-2 geborgen. Die Kerne bestanden aus $1 \mathrm{~m}$ langen Abschnitten und deckten folgende Tiefen ab: 0-100 cm, $100-200 \mathrm{~cm}$ und 200-300 cm für GR-6 sowie 38-138 cm und 138-238 cm für GR-7. Nachdem die Kerne anhand lithostratigraphischer Merkmale korreliert werden konnten, wurden sie durchgehend in $0.5 \mathrm{~cm}$ breite Scheiben mit einem Volumen von $22 \mathrm{~cm}^{3}$ geschnitten. Die kontinuierlichen Makrorestproben wurden in folgenden Tiefen entnommen: $224-189 \mathrm{~cm}$ in GR-7, 194-168.5 cm in GR-6, 177-132.5 cm in GR-7, $138-116 \mathrm{~cm}$ in GR-6. Die Überlappungen dienten der Überprüfung einer allfälligen biostratigraphischen Variation in den zwei Kernen. Die Sedimentscheiben wurden in einem Sieb von $0.2 \mathrm{~mm}$ Maschenweite ausgeschlämmt (Lang 1994). Die grösseren Holzstücke wurden nach dem Bestimmungsschlüssel von Schweingruber (1990) bestimmt. Die übrigen Pflanzenreste wurden mit Hilfe der Rezentmakrorestsammlung und unveröffentlichten Unterlagen des Instituts für Pflanzenwissenschaften der Universität Bern, der Vergleichsbeispiele aus dem Botanischen Garten und dem Untersuchungsgebiet sowie der Bestimmungsbücher von Katz et al. (1965), Schoch et al. (1988) und Godet (1998) bestimmt.

Das Makrorestdiagramm wurde mithilfe der Programme TILIA und TILIA * GRAPH als Konzentrationsdiagramm berechnet und erstellt (Grimm 1992). Dabei wurde unterschieden zwischen Makrorestflächen (Holz und Periderm) in $\mathrm{mm}^{2}$ pro 
$22 \mathrm{~cm}^{3}$ Sediment und Makroreststücken (Nadeln, Blätter, Samen usw.) pro $22 \mathrm{~cm}^{3}$ Sediment. Für die Berechnung der Prozentwerte wurden nur die Makroreststücke derjenigen Taxa berücksichtigt, welche eindeutig Bäumen (kennzeichnend für Wälder), Sträuchern und Zwergsträuchern (Wald- und Baumgrenzökoton) oder Spaliersträuchern und Kräutern (alpine Rasen, Geröllhalden, Schneetälchen) zugeordnet werden konnten. Wasserpflanzen, Cyperaceae und Makrorestflächen wurden von der Makrorestsumme ausgeschlossen (Tinner und Theurillat 2003).

Zur Radiokarbondatierung wurden 21 pflanzliche Makroreste terrestrischen Ursprungs verwendet. Die Datierungen der Proben wurden nach der AMS-Methode (accelerator mass spectrometry) im R. J. Van de Graaff Laboratorium der Universität Utrecht durchgeführt. Die Radiokarbonalter wurden mit dem Programm calib rev4.1.2 (Stuiver et al. 1998) kalibriert und in kalibrierten Jahre vor heute (cal. BP) ausgedrückt. Für den Vergleich zwischen GR-2 und GR-6/7 wurden die Tiefen von GR-2 anhand der Lithostratigraphie (beispielsweise Siltschichten) auf die Tiefenskala von GR-6/7 übertragen.

Um die Reproduzierbarkeit der Ergebnisse zu prüfen, wurde die Biostratigraphie der Makrorestkonzentrationen bzw. -flächen dieser Arbeit (GR-6/7) mit derjenigen aus GR-2 verglichen. Die Proben beider Arbeiten wiesen mit $25 \mathrm{~cm}^{3}$ (GR-2) bzw. $22 \mathrm{~cm}^{3}$ (GR-6/7) ein vergleichbares Volumen auf. Da in der vorliegenden Arbeit die Makroreste in einer höheren Auflösung (alle $0.5 \mathrm{~cm}$ ) als bei der ersten Untersuchung (Spätglazial alle $5 \mathrm{~cm}$; Holozän alle $3 \mathrm{~cm}$ ) gezählt wurden, wurden als Annäherung an GR-2 durch Zusammenlegen neue Proben von $3 \mathrm{~cm}$ Umfang sowie einem Volumen von $25 \mathrm{~cm}^{3}$ berechnet.

\section{Resultate und Interpretation}

Chronologie

Von den 21 datierten Proben konnten 12 für die Chronologie verwendet werden, während neun Datierungen aufgrund des biostratigraphischen Vergleichs mit dem Makrorestdiagramm GR-2 aus Wick und Tinner (1997) sowie Vergleichen innerhalb dieser Arbeit zu jung erschienen (Tab. 1, Abb. 1). Die Ursache hierfür konnten wir leider nicht in Erfahrung bringen; Parallelisierungsschwierigkeiten konnten ausgeschlossen werden. Aufgrund der mit Sicherheit zu jungen Altersangabe der Basisprobe aus einer Tiefe von $222 \mathrm{~cm}$ wurde das Alter-Tiefen-Diagramm (Abb. 1) ab $209 \mathrm{~cm}$ Tiefe mithilfe der Datierungen des GR-2 aus Tinner et al. (1996) linear verlängert. Durch lineare Interpolation der kalibrierten ${ }^{14} \mathrm{C}$ Daten wurde die Alters-Tiefen-Kurve des Gouillé Rion dargestellt.

Lokale Makrorest-Diagramm-Abschnitte und vegetationsgeschichtliche Entwicklung

Das Makrorestdiagramm wurde in acht lokale Makrorestzonen (local macrofossil assemblage zones, LMAZ) mit annähernd einheitlicher Zusammensetzung unterteilt (Abb. 2). Diese werden nachfolgend kurz beschrieben und bezüglich der vorkommenden Vegetation interpretiert.

Rn-1 Kräuter-Zone (224-204 cm; 12'000-11'000 cal. BP)

Carex-Früchte; ab $222 \mathrm{~cm}$ Salix herbacea-Blätter, -Knospen und -Holz; ab $214 \mathrm{~cm}$ vereinzelte Samen verschiedener Kräuter (Saxifraga cuneifolia T., Potentilla argentea T., Rumex); $208 \mathrm{~cm}$ erste Larix decidua-Nadel; $206 \mathrm{~cm}$ erstes Dryas octopetala-Blatt 
Tab. 1. AMS-Radiokarbon-Datierungen des Gouillé Rion (GR-6 und 7).

\begin{tabular}{|c|c|c|c|}
\hline Kern & Material & Tiefe $(\mathrm{cm})$ & $\begin{array}{l}{ }^{14} \mathrm{C}-\mathrm{Alter} \\
\text { (BP conv.) }\end{array}$ \\
\hline GR-6 & Juniperus nana Nadel, indet. Blatt & $120.5-120$ & $2 ’ 827 \pm 32$ \\
\hline \multirow[t]{2}{*}{ GR-6 } & Juniperus nana Nadel, Empetrum & & \\
\hline & hermaphroditum Blatt, indet. Periderm & $131-130.5$ & $3^{\prime} 541 \pm 44$ \\
\hline GR-7* & Pinus cembra Blütenstand & $139-138.5$ & $3^{\prime} 460 \pm 42$ \\
\hline GR-7 & indet. Periderm & $144.5-144$ & $4^{\prime} 285 \pm 41$ \\
\hline GR-7* & Pinus cembra Blütenstand, -Nadeln, -Kurztrieb & $147-146.5$ & $4 ’ 284 \pm 47$ \\
\hline GR-7* & Pinus cembra Blütenstand, -Nadeln, -Kurztrieb & $149-148.5$ & $4{ }^{\prime} 330 \pm 50$ \\
\hline GR-7 & Koniferen-Periderm & $155-154.5$ & $5 ’ 244 \pm 50$ \\
\hline GR-7 & Pinus cembra Holz & $159.5-159$ & $5^{\prime} 670 \pm 50$ \\
\hline GR-7 & Koniferen Periderm & $163.5-163$ & $6 ’ 026 \pm 49$ \\
\hline GR-7* & Pinus cembra Kurztriebe, -Nadeln & $166-165.5$ & $6{ }^{\prime} 040 \pm 50$ \\
\hline GR-7* & Pinus cembra Kurztriebe, -Nadeln & $168-167.5$ & $6{ }^{\prime} 120 \pm 50$ \\
\hline GR-7 & indet. Holz & $173.5-173$ & $7 ’ 100 \pm 60$ \\
\hline GR-6* & Pinus cembra Holz & 174-173.5 & $6{ }^{\prime} 900 \pm 70$ \\
\hline GR-6 & Pinus cembra Nuss & $179-178.5$ & $7^{\prime} 630 \pm 60$ \\
\hline GR-6 & Laubgehölz Periderm & $184-183.5$ & $8^{\prime} 080 \pm 60$ \\
\hline \multirow[t]{2}{*}{ GR-6* } & Pinus cembra Kurztrieb, -Nadeln, & & \\
\hline & Koniferen Holz & $189-188.5$ & $7^{\prime} 400 \pm 50$ \\
\hline GR-7 & Pinus cembra Holz, -Periderm & $193-192.5$ & $8 ' 450 \pm 70$ \\
\hline GR-7 & Larix decidua Blütenstände, - Holz & $200-199.5$ & $9^{\prime} 080 \pm 70$ \\
\hline \multirow[t]{2}{*}{ GR-7* } & Dryas octopetala Blätter, Larix decidua & & \\
\hline & Nadeln & 204-203.5 & $8^{\prime} 870 \pm 90$ \\
\hline GR-7 & $\begin{array}{l}\text { Larix decidua Nadeln, Salix herbacea Blatt, } \\
\text { indet. Holz }\end{array}$ & 209-206 & $10^{\prime} 100 \pm 80$ \\
\hline GR-6* & Salix herbacea Blätter & $222.5-222$ & $8 ' 480 \pm 70$ \\
\hline
\end{tabular}

*verworfene Datierungen

Das spärliche Auftreten einzelner Kräuterblätter und -samen lässt von 12'000-11'000 cal. BP zuerst auf lockere, dann auf dichtere alpine Rasen rund um den Gouillé Rion schliessen. Die Funde von Lärchennadeln gegen Ende der Zone deuten darauf hin, dass sich einzelne Lärchen nicht mehr sehr weit unterhalb des Seeleins befinden. Bei der Interpretation gilt allerdings zu beachten, dass die Lärchennadeln aufgrund der offenen Vegetation relativ weit transportiert werden konnten, so dass der Abstand der Waldgrenze noch bis zu 100-200 m betragen konnte (s. Profil Lengi Egga, Tinner und Theurillat, 2003).

Rn-2 Larix decidua-Salix-Dryas octopetala-Juniperus nana-Zone (204-200 cm; 11’000-10’250 cal. BP)

Werte der L. decidua-Nadeln ansteigend, kontinuierlich; $202.5 \mathrm{~cm}$ erste Pinus cembraNadel; bis 202.5 cm Salix (Holz, Knospen); D. octopetala Maximum; Juniperus nana Anstieg; einzelne Betula pendula/pubescens-Früchte sowie Rumex-Samen; je ein Blatt von Soldanella sowie ein Same von Caryophyllaceae 


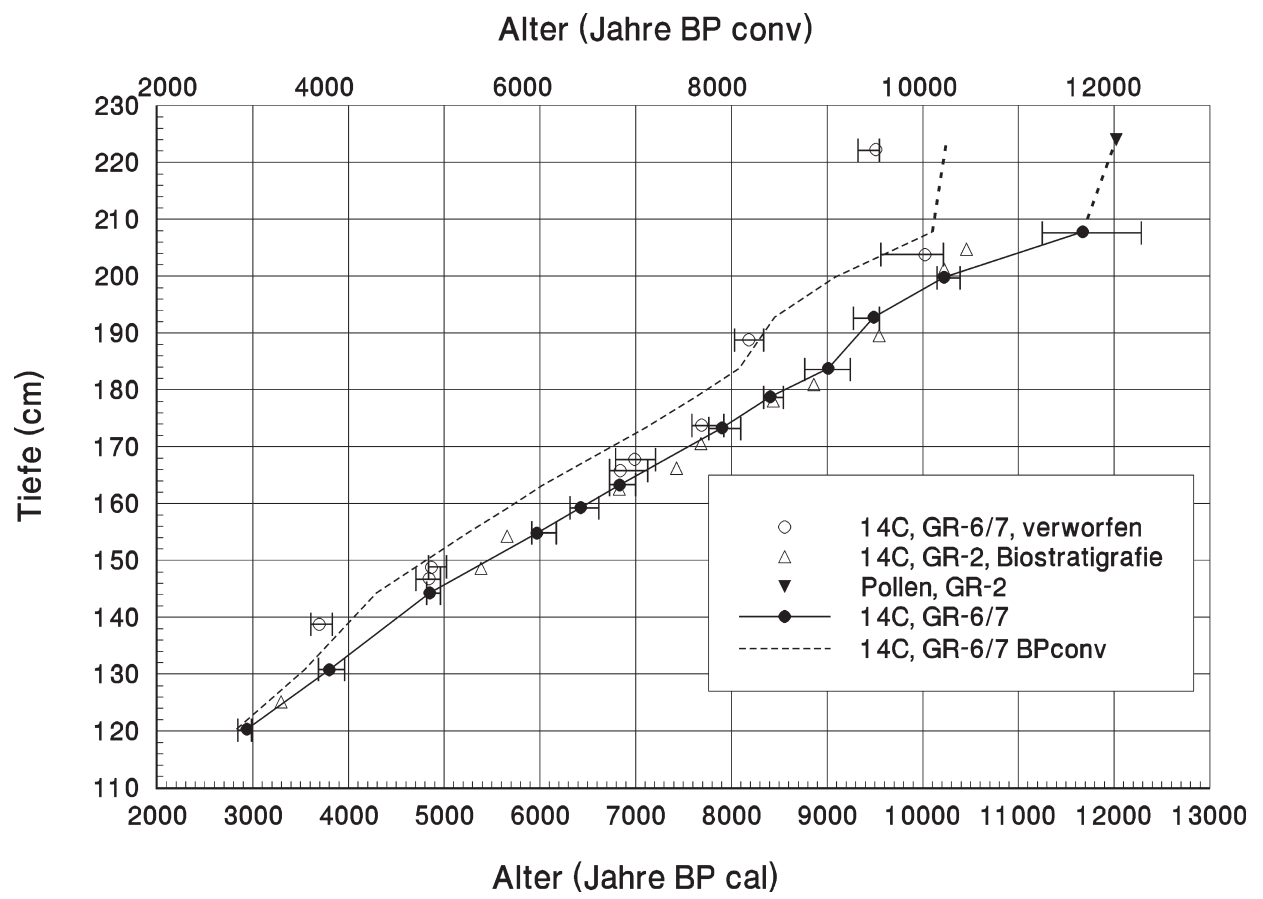

Abb. 1. Radiokarbondatierungen der Sedimente aus den Kernen GR-6/7 des Gouillé Rion. Die waagrechten Balken zeigen das $95 \%$ Konfidenzintervall der kalibrierten Alter. Als Vergleich sind ebenfalls die Daten aus dem Kern GR-2, Biostratigraphie abgebildet. Für den Vergleich zwischen GR-2 und GR-6/7 wurden die Tiefen von GR-2 anhand der Lithostratigraphie (beispielsweise Siltschichten) auf die Tiefenskala von GR-6/7 übertragen.

Um 10'700 cal. BP breitete sich die lichtliebende Lärche als Pionier auf der Hochebene der Alpe d'Essertse aus, was auf die allgemeine Aufwärtsverschiebung der Baum- und Waldgrenzen im Präboreal hinweist (Lang 1994). Ein möglicher Grund für die schnellere Besiedlung durch die Lärche gegenüber der Arve ist die Windverbreitung der leichten Lärchensamen im Gegensatz zur Tierverbreitung der schwereren Arvensamen (Ellenberg 1996). Ungefähr gleichzeitig mit L. decidua erreichen zu Beginn dieser Zone die ersten J. nana-Zwergsträucher das Gebiet. Vorerst herrscht jedoch noch eine andere Zwergstrauchart, die sehr lichtbedürftige $D$. octopetala, zusammen mit Salix vor. Diese zwei Taxa bilden mit den offenen L. decidua-Beständen den zur damaligen Zeit engen Übergangsbereich zwischen der subalpinen und der alpinen Stufe (Tinner und Theurillat 2003). Das Landschaftsbild dürfte um 11'000-10'250 cal. BP somit folgendermassen aussehen: Der Gouillé Rion liegt nahe der Waldgrenze; Vorherrschend sind offene L. decidua-Bestände mit einzelnen Pinus cembra. Diese Bestände sind mosaikartig von J. nana-Zwergsträuchern durchsetzt.

Rn-3 Larix decidua-Pinus cembra-Juniperus nana-Zone (200-194 cm;10'250-9'600 cal. BP) 


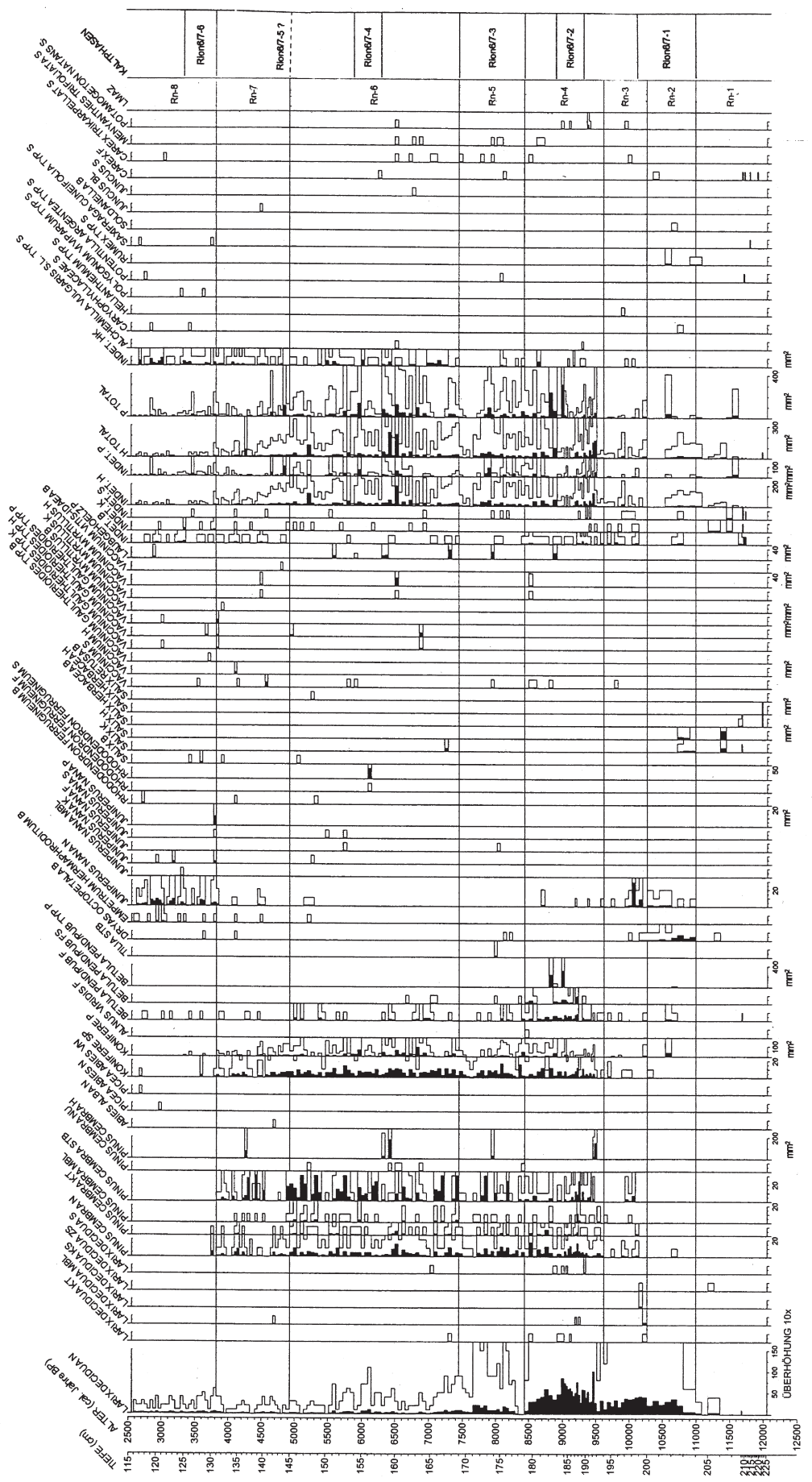

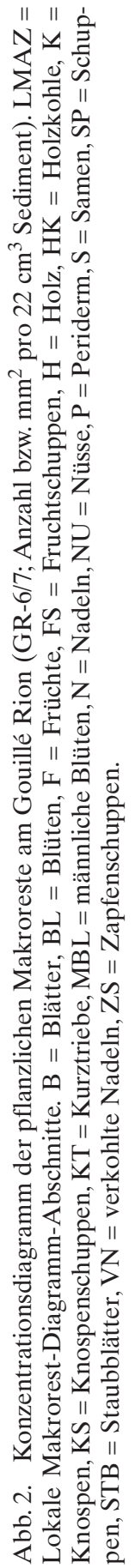


L. decidua Nadeln Gipfel; verschiedene andere Reste von L. decidua (Periderm, Kurztrieb, männliche Blüten etc); J. nana Maximum und Rückgang; verschiedene Teile von $P$. cembra; D. octopetala nur noch vereinzelt anfangs dieser Zone; $196 \mathrm{~cm}$ erster Vaccinium Rest (Same); $197 \mathrm{~cm}$ einziger Fund eines Helianthemum Samens; vermehrt nicht näher bestimmte Blätter; in zwei Tiefen, 198.5 und 197.5 cm, sehr geringe Fläche Holzkohle; $197.5 \mathrm{~cm}$ erster Potamogeton natans Same

Die offenen Bestände schliessen sich allmählich zu einem geschlossenen Wald. Dies zeigt neben den hohen Makrorestkonzentrationen verschiedener Teile der zwei Koniferenarten (Nadeln, Kurztriebe, männliche Blüten, Samen, Koniferenperiderm usw.) auch das Verschwinden der zwei lichtliebenden Zwergstraucharten J. nana und D. octopetala am Ende der Zone. Am Anfang der Zone verdrängt J. nana als etwas grösserer, mehr Schatten ertragender Zwergstrauch, die lichtliebenden Bodenkriecher D. octopetala und Salix sp. (cf. herbacea).

Rn-4 Pinus cembra-Larix decidua-Betula pendula/pubescens-Zone (194-178 cm; 9'600-8'350 cal. BP)

Maxima von L. decidua (Nadeln), P. cembra (Nadeln) und B. pend./pub. (Früchte, Fruchtschuppen, Periderm); L. decidua Samen und männliche Blüten; Übergang Rn-4 zu Rn-5 starker Rückgang von Larix decidua Nadeln; in dieser und den drei folgenden Zonen häufige Funde von P. cembra männliche Blüten, $179 \mathrm{~cm}$ Nuss derselben Art; J. nana nur noch vereinzelt; Vaccinium (Same) und V. myrtillus (Knospe, Holz) vereinzelt; Maxima von nicht näher bestimmten Holz- und Peridermstücken; $179.5 \mathrm{~cm}$ einziger Fund einer Alnus viridis Frucht; vermehrt nicht näher bestimmte Blätter; vereinzelt $P$. natans; $181.5 \mathrm{~cm}$ erster Menyanthes trifoliata Same; vereinzelte Holzkohle-Funde

Mit der Zunahme der P. cembra- und L. decidua-Makroreste in dieser Zone steigt auch die Konzentration der geflügelten Früchte und Fruchtschuppen der lichtliebenden Baumbirke (B. pendulalpubescens). Diese leichten, vom Wind verbreiteten Teile könnten von Gebüschen stammen, welche auf den Felsen oberhalb des Seeleins gewachsen sind; solche strauchförmig wachsenden Birken (B. pendula) kommen heute noch auf der Staumauer vor. Der spätere Fund eines grossen Peridermstückes um 9'000 cal. BP sowie etliche Holz- und Peridermstücke von Laubgehölzen des Types Betula setzt aber das Vorkommen der Birke auch rund um den See voraus. Auch am Hobschensee (2'017 m) werden von Lang und Tobolski (1985) die subalpinen Wälder mit L. decidua, P. cembra und B. pend./pub. beschrieben. Ob es sich bei den Birken in der Nähe des Gouillé Rion auch um grosse Bäume handelte, bleibt jedoch fraglich.

Rn-5 Larix decidua-Pinus cembra-Zone (178-169 cm; 8’350-7’450 cal. BP)

L. decidua-Nadeln tiefere Werte als in der vorigen Zone und keine Samen und männliche Blüten mehr; P. cembra gleichbleibend; B. pend./pub. nur noch vereinzelt; Übergang Rn-5 zu Rn-6 allgemeiner Rückgang von L. decidua, P. cembra und B. pend./pub.; $174.5 \mathrm{~cm}$ einziger Fund zweier Tilia Antheren; vereinzelt D. octopetala Blätter; $175 \mathrm{~cm}$ Frucht von J. nana; vereinzelte Holzkohle-Funde

V. myrtillus (Holz, Knospen) ist als typische Unterwuchsart des subalpinen Lärchen-Arvenwaldes durch Holz und Knospen belegt, welche mit Sicherheit nicht weit von ihrem Wuchsort abgelagert wurden. Da L. decidua ihre Nadeln jeden Herbst abwirft, muss die Anzahl der gefundenen Nadeln deutlich weniger gewichtet werden 
als diejenige der langlebigeren P. cembra Nadeln. Obwohl in dieser Zone pro Probe durchschnittlich 11 Lärchennadeln und bloss fünf Arvennadeln gefunden wurden, muss man deshalb annehmen, dass im Gebiet nach 8'400 cal. BP P. cembra die dominante Baumart nahe der Waldgrenze wurde. Ein weiteres Indiz für einen dichten Arvenwald nach 8'400 cal. BP ist das nur noch spärliche Vorkommen von Baumbirkenresten.

Rn-6 Pinus cembra-Zone (169-145 cm; 7'450-4'950 cal. BP)

L. decidua fast durchgehend, jedoch tiefere Werte als in der vorigen Zone; P. cembra Nadeln sowie Kurztriebe gleichbleibend wie vor Rückgang Ende der vorigen Zone, vermehrt männliche Blüten und Nüsse; Maximum Koniferenperiderm; B. pend./pub. und $J$. nana vereinzelt; erste Reste von Vaccinium gaultherioides (Holz, Knospen) und V. vitisidaea (Blatt); verschiedene Teile von Rhododendron ferrugineum; $148 \mathrm{~cm}$ erstes Blatt von Empetrum hermaphroditum; $160.5 \mathrm{~cm}$ letzte Samen von M. trifoliata und P. natans; häufige Holzkohle-Funde

Es herrscht im Allgemeinen ein stabiler subalpiner Arvenwald mit einigen Lärchen vor. Die Schwankungen in der Makrorest-Konzentrationskurve (beispielsweise gerade anfangs dieser Zone) dürften auf Kaltphasen zurückzuführen sein (siehe Diskussion). Die Zunahme der makroskopischen Holzkohleflächen zu Beginn dieser Zone bis hin zum oberen Ende des Sediments um 2'500 cal. BP ist als Zunahme von Waldbränden zu deuten. Im Unterholz gedeihen Vaccinium sowie R. ferrugineum Zwergsträucher.

Rn-7 Pinus cembra-Vaccinium-Zone (145-131 cm; 4'950-3'800 cal. BP)

L. decidua Nadeln tiefere Werte als in der vorangegangenen Zone; P. cembra Nadeln nicht mehr kontinuierlich und tiefere Werte; P. cembra Kurztriebe und männliche Blüten tiefere Werte und keine Nüsse mehr; $142.5 \mathrm{~cm}$ einziger Fund einer Abies alba Nadel; $B$. pend./pub. Rückgang; Abnahme nicht näher bestimmter Holz- und Peridermstücke; Teile von Vaccinium/V. myrtillus etwas häufiger als in voriger Zone; E. hermaphroditum und $J$. nana vereinzelt; einzelnes Blatt von $D$. octopetala und $R$. ferrugineum; vermehrt nicht näher bestimmte Blätter; fast kontinuierliche Holzkohle Funde

Wichtigstes Merkmal dieser Zone ist der langsame Rückzug der Arve, was gleichbedeutend mit einer beginnenden Absenkung der Waldgrenze ist. Nachdem um den Gouillé Rion während rund 3'500 Jahren ein geschlossener Arvenwald bestanden hatte, reduzierte sich das Vorkommen von $P$. cembra auf einzelne Bäume oder lockere Bestände. Dasselbe gilt für L. decidua; von ihr waren ebenfalls Einzelbäume vorhanden, sie nimmt auch nach der Abnahme der Arve nicht mehr zu. Das vereinzelte Auftreten von Blättern der Zwergsträucher E. hermaphroditum und D. octopetala sowie von nicht näher bestimmten Kräutern zeigt ebenfalls die Veränderung der Vegetationszusammensetzung von Wald in subalpine Zwergstrauchheiden und Rasen.

Rn-8 Juniperus nana-Empetrum hermaphroditum-Zone (131-116 cm; 3'800-2'600 cal. BP) L. decidua Nadeln gleichbleibend; letzte P. cembra Reste (fünf Nadeln, ein Kurztrieb) bei $130.5 \mathrm{~cm}$ (3'800 cal. BP); in $121 \mathrm{~cm}$ (3'000 cal. BP) sowie $117.5 \mathrm{~cm}$ (2'700 cal. BP); Nadel bzw. verkohlte Nadel von Picea abies; B. pend./pub. Früchte vereinzelt; J. nana allgemein (Nadeln, Knospen, Samen) höhere Werte, Nadeln fast kontiniuerlich, männliche Blüten und Periderm nur in dieser Zone vorkommend; E. hermaphroditum Höchststand; einzelnes Blatt von $D$. octopetala sowie $R$. ferrugineum; nicht näher bestimmte Blätter gleich- 
bleibend; vereinzelt Teile von Vaccinium/V. gaultherioides; allgemeines Maximum an Samen verschiedener Kräuter (Caryophyllaceae, Polygonum viviparum T., Potentilla argentea T., Saxifraga cuneifolia T.) sowie Holzkohle

Nach 3'750 cal. BP ist die Arve vollständig aus dem Gebiet verschwunden, nur einige Lärchen stehen noch nahe des Seeleins. Die einzelne Nadel von Picea abies um 3'000 cal. BP beweist, dass zu dieser Zeit die Rottanne nicht weit unterhalb des Gouillé Rion gewachsen sein muss. Ihre heutige Obergrenze beträgt im Gebiet ca 2'100 m. Im Makrorestdiagramm vorherrschend ist in dieser letzten Zone J. nana zusammen mit E. hermaphroditum. Diese zwei Zwergstraucharten bilden zusammen mit $R$. ferrugineum, Vaccinium und D. octopetala den Übergangsbereich von der subalpinen zur alpinen Stufe, welcher laut Ellenberg (1996) in dieser Zusammensetzung als anthropo-zoogen angesehen werden muss.

Vergleich der Biostratigraphien

Abbildung 3 zeigt, dass die Biostratigraphie von GR-2 weitgehend reproduziert werden konnte, vor allem anhand der Nadelkurven der wichtigsten waldgrenzbildenden Baumarten Pinus cembra und Larix decidua. Im Kern GR-2 sind in den Tiefen 186-183 cm sowie 177-174 cm ganze Juniperus nana Zweiglein gefunden worden, was die höheren Nadel-Konzentrationen gegenüber GR-6/7 erklärt. Die Absolutwerte der Holz- bzw. Peridermflächen der beiden Studien stimmten weniger gut überein, da es sich hierbei meist um ganze Zweiglein mit mehreren $\mathrm{mm}^{2}$ Fläche handelt, so dass die Heterogenität der Verteilung grösser ist.

\section{Diskussion}

Reproduzierbarkeit der Biostratigraphien vom Gouillé Rion

Aus Abbildung 3 und Tabelle 2 geht hervor, dass sich die Vegetationstypen aus der vorliegenden Arbeit und aus derjenigen von Tinner et al. (1996) relativ gut entsprechen. Die höhere zeitliche Auflösung sowie die genauere Bestimmung zahlreicherer Makrorestteile in der vorliegenden Arbeit ermöglichte zusätzlich eine feinere Unterteilung der Vegetationstypen wie auch eine genauere zeitliche Einteilung der Vegetationsgeschichte.

Schwierigkeiten beim Vergleich mit GR-2 bestanden erstens aufgrund der Chronologie, da Unschärfen wegen den ${ }^{14} \mathrm{C}$ Datierungen unvermeidbar sind. Weiter waren mehrere Fehldatierungen zu verzeichnen. Zweitens konnten einzelne Kurven, welche auf kleinen Summen beruhen (z.B. Pinus cembra Nadeln), weniger genau reproduziert werden als solche, die auf grösseren Summen beruhen (z.B. Larix decidua Nadeln). Diese Schwierigkeiten könnten durch eine Pollenanalyse in den Kernen GR-6/7 behoben werden, da Pollen meistens höhere Summen, d.h. statistisch zuverlässigere Werte liefern als Makroreste. Die Pollendaten würden es somit erlauben, die Kaltphasen am Gouillé Rion besser zu präzisieren.

Die Ergebnisse dieser Arbeit haben jedoch auch deutlich gezeigt, welche Vorteile die Analyse von pflanzlichen Makroresten zur Rekonstruktion früherer Baum- und Waldgrenzen gegenüber Pollenstudien hat (Birks 2001; Tinner und Theurillat 2003). Die taxonomisch hohe Auflösung (Gattungsebene, oft bis auf die Artebene) erlaubte beispielsweise die Unterscheidung der Zwergsträucher Vaccinium sp. bzw. V. myrtillus,V. gaultherioides und V. vitis-idaea, während diese anhand der Pollen alle zusammen 


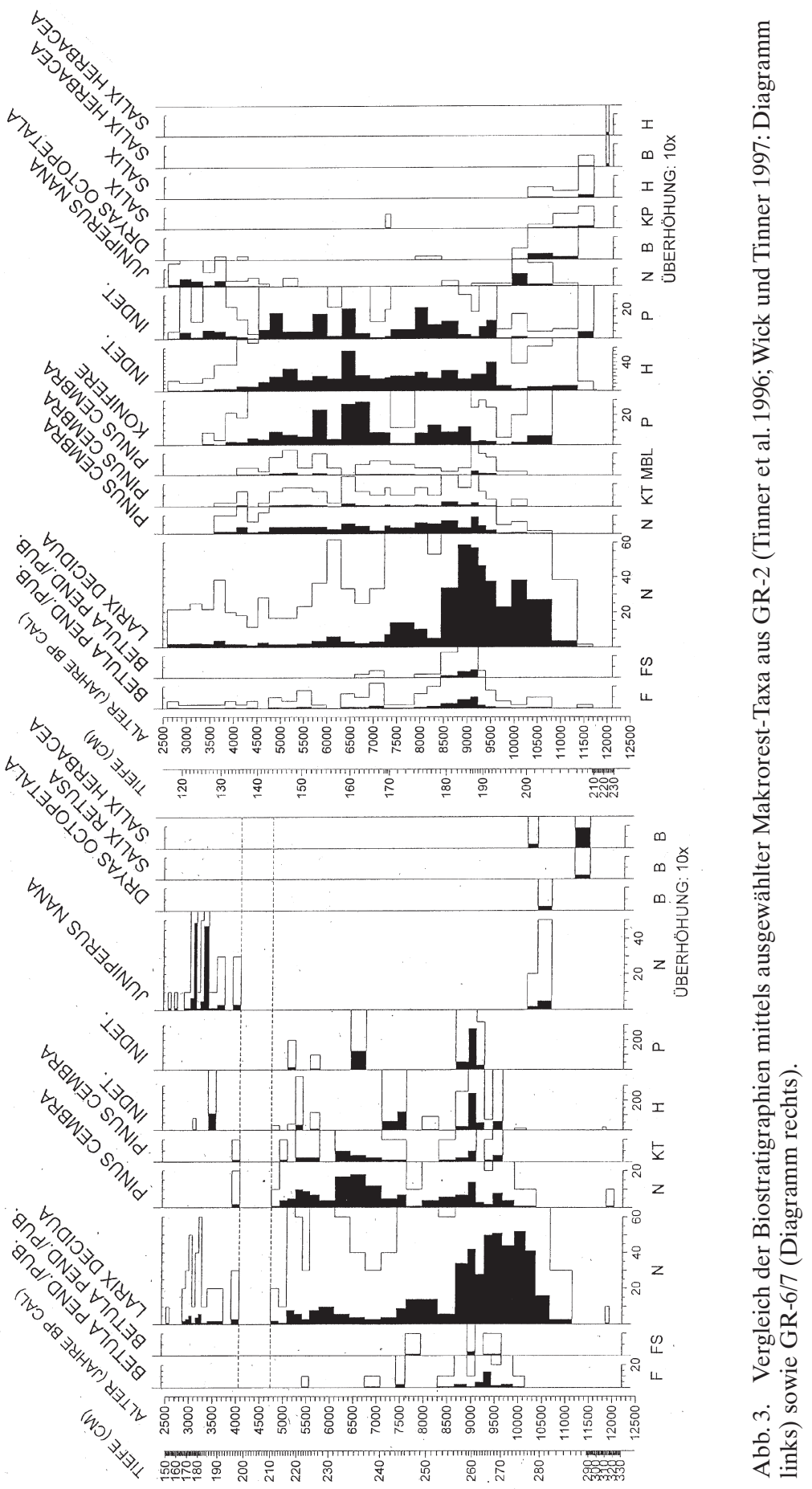


Tab. 2. Vergleichende Biostratigraphie des Gouillé Rion aufgrund der Kerne GR-6/7 (Makroreste, aktuelle Arbeit) und GR-2 (Pollen und Makroreste, Tinner et al. 1996) mit Angabe der Zonen, der rekonstruierten Vegetationstypen und dem ${ }^{14} \mathrm{C}$ Alter in cal. BP.

\begin{tabular}{|c|c|c|c|c|c|}
\hline \multicolumn{3}{|c|}{ Kerne GR-6/7 } & \multicolumn{3}{|c|}{ Kern GR-2 } \\
\hline $\begin{array}{l}\text { Zone } \\
\text { (LMAZ) }\end{array}$ & Vegetation & Alter (cal. BP) & Zone* & Vegetation & Alter (cal. BP) \\
\hline $\mathrm{Rn}-8$ & $\begin{array}{l}\text { Zwergwachol- } \\
\text { derheiden mit } \\
\text { einzelnen } \\
\text { Lärchen }\end{array}$ & 3'800-2'600 & $\begin{array}{l}\text { PAZ Ri-6 } \\
\text { MZ Rn-5 }\end{array}$ & $\begin{array}{l}\text { Zwergwachol- } \\
\text { derheiden mit } \\
\text { Grünerlenge- } \\
\text { büschen und } \\
\text { einzelnen Lär. }\end{array}$ & 3'850-2'800 \\
\hline $\mathrm{Rn}-7$ & $\begin{array}{l}\text { Offene Arven- } \\
\text { bestände mit } \\
\text { einzelnen } \\
\text { Lärchen }\end{array}$ & $4^{\prime} 950-3^{\prime} 800$ & \multirow[t]{2}{*}{$\begin{array}{l}\text { PAZ Ri-5 } \\
\text { MZ Rn-4 }\end{array}$} & \multirow{2}{*}{$\begin{array}{l}\text { Rückgängiger } \\
\text { Arvenwald mit } \\
\text { einzelnen } \\
\text { Lärchen } \\
\text { Arvenwald mit } \\
\text { einzelnen } \\
\text { Lärchen }\end{array}$} & \multirow{2}{*}{ 4'950-3'850 } \\
\hline \multirow[t]{2}{*}{$\mathrm{Rn}-6$} & \multirow[t]{2}{*}{$\begin{array}{l}\text { Arvenwald mit } \\
\text { einzelnen } \\
\text { Lärchen }\end{array}$} & \multirow[t]{2}{*}{ 7'450-4'950 } & & & \\
\hline & & & & Wald mit & $10 ’ 250-6 ’ 600$ \\
\hline $\mathrm{Rn}-5$ & $\begin{array}{l}\text { Lärchen-Arven- } \\
\text { wald }\end{array}$ & $8^{\prime} 350-7^{\prime} 450$ & & $\begin{array}{l}\text { Lärchen und } \\
\text { zunehmender } \\
\text { Arven-Domi- } \\
\text { nanz sowie } B \text {. } \\
\text { pendula/pub. }\end{array}$ & \\
\hline $\mathrm{Rn}-4$ & $\begin{array}{l}\text { Lärchen-Arven- } \\
\text { wald mit Betula } \\
\text { pendula/pub. }\end{array}$ & $9^{\prime} 600-8{ }^{\prime} 350$ & & & \\
\hline $\mathrm{Rn}-3$ & $\begin{array}{l}\text { Offene Lär- } \\
\text { chenbestände } \\
\text { mit einzelnen } \\
\text { Arven und } \\
\text { J. nana }\end{array}$ & $10 ’ 250-9 ' 600$ & & & \\
\hline $\mathrm{Rn}-2$ & $\begin{array}{l}\text { Offene Lär- } \\
\text { chenbestände } \\
\text { mit Salix, D.oc- } \\
\text { topetala und } \\
\text { wenig } J . \text { nana }\end{array}$ & $11^{\prime} 000-10^{\prime} 250$ & $\begin{array}{l}\text { PAZ Ri-4 } \\
\text { MZ Rn-3 }\end{array}$ & $\begin{array}{l}\text { Offene Lär- } \\
\text { chenbestände } \\
\text { mit D. octo- } \\
\text { petala und } \\
\text { J. nana }\end{array}$ & $11^{\prime} 000-10 ’ 250$ \\
\hline Rn-1 & $\begin{array}{l}\text { Alpine Rasen, } \\
\text { Pioniervege- } \\
\text { tation }\end{array}$ & $12^{\prime} 000-11^{\prime} 000$ & $\begin{array}{l}\text { PAZ Ri-3 } \\
\text { MZ Rn-2 }\end{array}$ & $\begin{array}{l}\text { Alpine Rasen, } \\
\text { Pioniervege- } \\
\text { tation }\end{array}$ & $12^{\prime} 900-11^{\prime} 000$ \\
\hline
\end{tabular}

* PAZ $=$ Pollen-Zone (pollen assemblage zone), MZ = Makrorest-Zone

auf Familienebene (Ericaceae) zu finden sind. Bei Salix konnte man einige Teile eindeutig den unter bzw. dicht am Boden kriechenden Spaliersträuchern Salix herbacea (Blätter, Holz) bzw. Salix retusa (Blätter) zuordnen. Bei der Gattung Juniperus zeigte 
Tab.3. Spätglaziale und holozäne Kaltphasen, die am Gouillé Rion (GR-6/7) anhand von Änderungen der Makrorest-Konzentrationen verschiedener Taxa festgestellt wurden, und Vergleich mit den rekonstruierten Kaltphasen in Zentraleuropa nach Haas et al. (1998). Die rekonstruierte Kaltphase CE-4 war am Gouillé Rion nicht ersichtlich.

\begin{tabular}{|c|c|c|c|}
\hline $\begin{array}{l}\text { Kaltphasen am } \\
\text { Gouillé Rion }\end{array}$ & $\begin{array}{l}{ }^{14} \text { C Alter } \\
\text { (cal. BP) }\end{array}$ & Makrorestkonzentrationen* & $\begin{array}{l}\text { Kaltphasen in } \\
\text { Zentraleuropa }\end{array}$ \\
\hline Rion 6/7-6 & 3'800-3’350 & $\downarrow$ P. cembra $; \uparrow J$. nana Nadeln & \multirow[t]{2}{*}{$\mathrm{CE}-7$} \\
\hline Rion 6/7-5? & 4'900-? & $\begin{array}{l}\downarrow \text { P. cembra Kurztriebe und } \\
\text { männliche Blüten; } \downarrow \text { Koniferen }\end{array}$ & \\
\hline & & Periderm; $\downarrow$ Holz & CE-6 \\
\hline Rion 6/7-4 & 6’300-5'900 & $\downarrow$ P. cembra; $\uparrow$ L. decidua & $\begin{array}{l}\text { CE-5 } \\
\text { CE-4 }\end{array}$ \\
\hline Rion 6/7-3 & $8^{\prime} 450-7^{\prime} 450$ & $\downarrow$ P. cembra; $\uparrow$ L. decidua & CE-3 \\
\hline Rion $6 / 7-2$ & 9'300-8'900 & $\uparrow$ Betula $; \downarrow$ P. cembra; $\uparrow$ L. decidua & $\mathrm{CE}-2$ \\
\hline Rion $6 / 7-1$ & $11^{\prime} 000-10 ’ 150$ & $\uparrow$ J. nana; $\uparrow$ D. octopetala & CE-1 \\
\hline
\end{tabular}

$* \uparrow$ : Zunahme und $\downarrow$ : Abnahme der Makrorest-Konzentrationen im Vergleich zu den Warmphasen

die Nadelform und -grösse, dass es sich um die subalpin/alpine Art J. nana handelt. Zudem sind diese und andere Arten (vor allem D. octopetala und L. decidua) im Pollendiagramm generell untervertreten. Die höhere taxonomische Auflösung mittels Makrorestanalyse erlaubt somit detailliertere Aussagen über frühere ökologische Zusammenhänge und die Vegetationsverhältnisse im Gebiet, beispielsweise konnte dadurch die Verdrängung der D. octopetala-Bestände durch J. nana während der Wiederbewaldungsphase nachgewiesen werden (Rn-3). Die Unterscheidung verschiedener Pflanzenteile (Abb. 2) ermöglichte Rückschlüsse über die räumliche Distanz zwischen den Ursprungs- und Ablagerungsorten. Beispielsweise fanden sich zuerst (Rn-1 bis Rn-3) nur die sehr leichten, geflügelten Früchte von B. pendula/pubescens; dies deutet darauf hin, dass diese aus grösserer Entfernung zum Gouillé Rion geweht wurden. In der Zone Rn-4 jedoch wurde die lokale Anwesenheit der Baumbirke zusätzlich durch die etwas schwereren Fruchtschuppen sowie Peridermstücke dieses Taxons in drei aufeinander folgenden Proben belegt. Von L. decidua zeugen in den Zonen Rn-1 und-2 nur Nadelfunde sowie ein Teil eines Zapfenschuppens, während von diesem Taxon in den darauffolgenden Zonen zusätzlich Kurztriebe, männliche Blüten, Knospenschuppen, Samen und Holz auftreten, was ebenfalls auf ein verstärktes lokales Vorkommen der Lärche im Gebiet hinweist.

Mittels Pollen- und Makrorestanalysen aus den Schweizer (Tinner et al. 1996) und italienischen Alpen (Wick 1994) wurden mehrere holozäne Warm- (Trocken-) und Kalt- (Feucht-) Phasen festgestellt (Wick und Tinner 1997). Die früher am Gouillé Rion (GR-2) ermittelten Kaltphasen Rion-1 bis Rion-7 wurden vorwiegend über die Pollenstratigraphie definiert (Wick und Tinner 1997). Im Sediment des Gouillé Rion bewirkten die holozänen Kaltphasen jeweils eine Auflockerung beziehungsweise einen leichten Rückgang der waldgrenzbildenden P. cembra. In der vorliegenden Studie wurden diese Kaltphasen vor allem anhand der Makrorest-Konzentrationen von P. cembra-, L. decidua- und J. nana-Nadeln, B. pendula/pubescens-Früchten und Fruchtschuppen wiedergefunden (Rion 6/7-1 bis Rion 6/7-6 in Abb. 2 und 3). Geringfügige Abweichungen sind mit den Datierungsschwierigkeiten (Tab. 1) zu erklären. Die in dieser 
Arbeit rekonstruierten Kaltphasen sind in Tabelle 3 dargestellt und ebenfalls in Abbildung 2 eingezeichnet. Die zweite Kaltphase während des Atlantikums, CE-4 (7'5007'100 cal. BP), ist in den Makroreststratigraphien des Gouillé Rion nicht ersichtlich. Haas et al. (1998) gehen davon aus, dass diese Abkühlung geringeres Ausmass hatte als die übrigen, was unser Ergebnis erklären könnte.

\section{Holozäne Waldgrenzschwankungen: klimatische und menschliche Einflüsse}

Zahlreiche in den Schweizer Zentralalpen durchgeführte Studien belegen, dass der moderne subalpine Vegetationsgürtel (1'800-2'400 m ü.M.) während der Jüngeren Dryas (12'600-11'550 cal. J BP) baumlos war (Markgraf 1969; Welten 1982; Lang und Tobolski 1985). Während dieser Kaltphase befand sich die obere Baumgrenze in den Zentralalpen auf ca. 1'500-1'600 m ü.M. (Welten 1982; Tobolski und Ammann 2000). Erstaunlich ist, dass am Gouillé Rion die erste Lärchennadel um 11'685 cal. BP, also gegen das Ende dieser Periode, gefunden wurde. So kann man sich vorstellen, dass während dieser Zeit die Baumgrenze im Gebiet unterhalb der Alpe d'Essertse etwas höher lag. Zur selben Zeit findet der Wechsel von anorganischem (Silt) zu vorwiegend organischem (Gyttja mit wenig Silt) Sediment statt. Weiter zeugen verschiedene pflanzliche Makroreste von Salix sowie Kräutern (indet. Blätter und Samen) von dichter werdenden Rasen. Die neuen Makrorestdaten zeigen also, dass sich die alpine Vegetation im Untersuchungsgebiet bereits ab 11'700-11'600 cal. BP erholt hat.

Die Phase der Wiederbewaldung mit Lärchen und Arven konnte in verschiedene Zonen (Rn-2 bis -6) aufgeteilt werden, in welchen zuerst offene Lärchenbestände mit einzelnen Arven, dann ein stabiler Lärchen-Arvenwald vorherrschte (Tab. 2). Diese Ablösung der Lärche durch die Arve entspricht der auch heute in subalpinen Wäldern beobachteten natürlichen Sukzession (Ellenberg 1996). Bemerkenswert ist jedoch, wie langsam die Sukzession im vorliegenden Fall verlaufen ist. Anhand des neuen Makrorestdiagramms nehmen wir an, dass erst nach 8'400 cal. BP P. cembra die dominante Baumart nahe der Waldgrenze wird. Dass die Arve ca 1'800 Jahre brauchte, um einen geschlossenen Baumbestand zu bilden, kann nicht einfach durch den Sukzessionsprozess erklärt werden, denn auf Gletschermoränen können Lärche und Arve innert 150 Jahren nach dem Eisrückzug dichte Wälder bilden (Ellenberg 1996). Demnach müssen weitere (insbesondere klimatische) Faktoren bei der langsamen Waldschliessung und Ausbreitung der Arve eine Rolle gespielt haben (Tinner und Kaltenrieder 2005). Mit der höheren zeitlichen Auflösung hatten wir gehofft, die hierfür vermutlich relevanten Kaltphasen zeitlich besser eingrenzen zu können, was aber aufgrund der chronologischen Unsicherheiten (Tab. 1) nur teilweise gelang (Abb. 2, Tab. 3).

Ab ca. 6'000 cal. BP (4'050 v. Chr.) zeigt eine Zunahme der Makrorestwerte von Sträuchern und Zwergsträuchern (Abb. 4) eine allgemeine Absenkung der Waldgrenze im Gebiet an. Wurde diese Absenkung der Waldgrenze bis auf den heutigen Stand, 2'100 m ü.M., vom Menschen oder vom Klima erwirkt oder spielten etwa beide Faktoren mit? Die Schwierigkeit in der Beantwortung der Frage besteht darin, dass anthropogen und klimatisch bedingte Veränderungen der Waldgrenzvegetation nur teilweise voneinander getrennt werden können (Ammann und Wick 1993).

Aus archäologischen Grabungen (Stöckli 1995) ist bekannt, dass um ca 7’450 cal. BP (ca 5'500 v. Chr.) in Sion erste Bauerngruppen siedelten. Pollenanalytische Studien im Walliser Rhonetal weisen Getreideanbau in der kollinen Stufe bereits für die Zeit um 7’000 cal. BP (5’050 v. Chr.) nach (Welten 1982). Die Frage stellt sich, wann der Mensch auch die höher gelegene Alpenstufe um den Gouillé Rion zu nutzen begann. Die bisherigen archäologischen Funde liefern keine direkten Beweise für das Vorhan- 


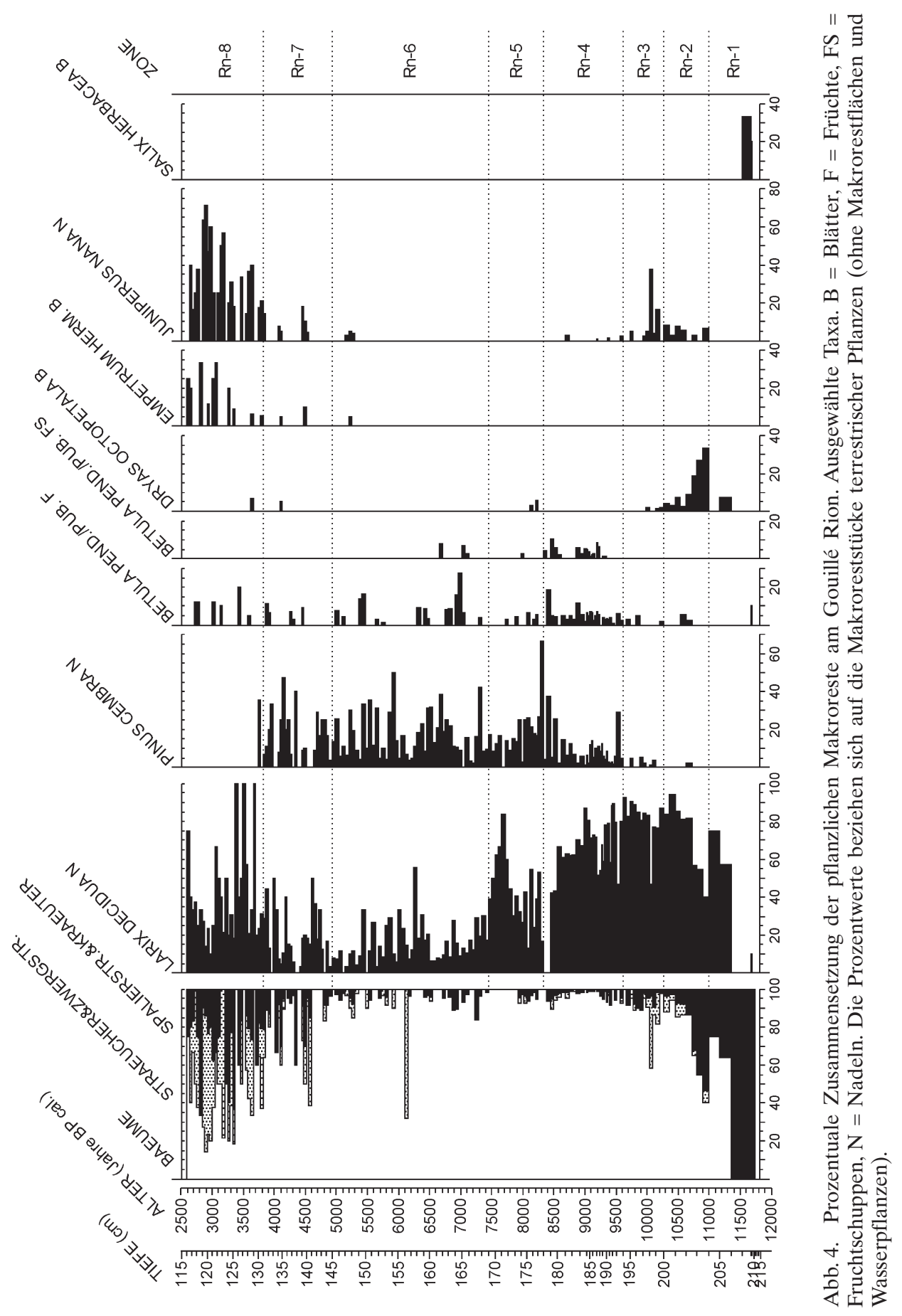


densein von Alpwirtschaft in der Region Hérémence und Alpe d'Essertse im frühen Neolithikum um 7'000 cal. BP (5'050 v. Chr.). Zahlreiche archäologische Funde in Felsabris im Gebiet des benachbarten Val d'Hérens und Zermatt zeigen aber, dass die Region bis in die alpine Stufe begangen wurde (Curdy et al. 1998). Baudais et al. (1990) halten fest, dass sich im 4. Jahrtausend v. Chr. (ab ca 6'000 cal. BP) der Getreideanbau nach archäologischen Hinweisen im heutigen Wallis in die untere subalpine Stufe ausbreitete.

Für das Gebiet um den Gouillé Rion ist im Pollendiagramm aus Tinner et al. (1996) ersichtlich, dass nach der Absenkung der Waldgrenze nach 5'500 cal. BP (3'550 v.Chr.) die Bestände langsam durch J. nana und Alnus viridis ersetzt werden. Nach den Autoren spricht einerseits das Auftreten von Kulturzeigerpollen wie Cannabaceae, Plantago lanceolata und Cerealia, und andererseits das gleichzeitige Ansteigen der Pollenkurve von Weidezeigern (vor allem Plantago alpina und J. nana) mit A. viridis für anthropogene Beeinflussung. Unterhalb der Alpe d'Essertse, in Vex (940 m ü.M.), wurden in archäologischen Grabungen aus der Früh- wie auch aus der Spätbronzezeit stammende Tierknochen gefunden, von denen 92\% Haus- und 8\% Wildtieren zugeordnet wurden (Chaix 1990). Das lässt auf einen Bedarf an Weideplätzen und Winterfutter in höheren Lagen schliessen, der zu weiteren Rodungen an der oberen Waldgrenze geführt haben dürfte (Aerni 1991). Für das Gebiet um den Gouillé Rion kann gemäss Tinner et al. (1996) geschlossen werden, dass das endgültige Verschwinden von P. cembra nach ca 3'750 cal. BP (1'800 v. Chr.) anthropogen verursacht wurde, indem die Bestände so geschwächt wurden, dass sie sich nach einer Kaltphase (Tinner et al. 1996: Rion-6 sowie Haas et al. 1998: CE-7) nicht mehr erholen konnten.

Da sich die Waldgrenze während des Holozäns auch dort nach unten verlagerte, wo menschlicher Einfluss zu dieser Zeit ausgeschlossen werden kann (z.B. Nordamerika, s. Bradley 1999), muss man annehmen, dass in erster Linie klimatische Faktoren zu dieser Entwicklung führten. Im Untersuchungsgebiet kann jedoch davon ausgegangen werden, dass sich dieser Effekt durch den menschlichen Einfluss verstärkt hat: Die Breite des anthropogen bedingten Waldgrenzökotons ist mittels Makroresttransekten auf ca 300-350 m geschätzt worden (Tinner und Theurillat 2003), während dieses von Natur aus ca. $100 \mathrm{~m}$ umfassen würde. Auch in der vorliegenden Studie zeigt die Zusammensetzung der obersten lokalen Makrorestzone (Rn-8), welche die Zeit nach der Absenkung der Waldgrenze darstellt, ein breites Baumgrenzökoton auf (Abb. 4). Im Makrorestdiagramm vorherrschend ist in dieser letzten Zone J. nana zusammen mit $E$. hermaphroditum. Die zwei Zwergstraucharten bilden zusammen mit $R$. ferrugineum, Vaccinium spp. und D. octopetala den Übergangsbereich von der subalpinen zur alpinen Stufe, welcher laut Ellenberg (1996) in dieser Zusammensetzung als anthropo-zoogen angesehen werden muss. Die vorliegende Untersuchung bestätigt also den starken menschlichen Einfluss auf die Waldgrenzentwicklung nach 6'000 cal. BP im Gebiet des Gouillé Rion.

\section{Schlussfolgerungen}

Die Reproduzierbarkeit der vegetationsgeschichtlichen Daten, welche aus Makroresten rekonstruiert wurden, war erstaunlich gut. Es hat sich herausgestellt, dass sowohl die allgemeinen Züge als auch manche Details (z.B. die Larix decidua Nadelkurve während des Holozäns) mehrheitlich korrekt wiedergegeben wurden. Die bisherigen Kenntnisse zu den Vegetationsveränderungen konnten in dieser Studie dank der hohen 
zeitlichen Auflösung und der Unterscheidung von Pflanzenteilen verfeinert werden. Insbesondere wurden die Vegetationsdynamik während der Wiederbewaldung und menschliche Einflüsse während der Absenkung der Waldgrenze genauer beschrieben.

Für Kommentare und Verbesserungsvorschläge zum Manuskript danken wir Dr. S. Güsewell, Dr. M. Schütz und Prof. Dr. S. Wegmüller.

\section{Literatur}

Aerni K. 1991. 1'000 Jahre Siedlung und Verkehr im schweizerischen Alpenraum. Voraussetzungen und Ergebnisse. In: Aerni K., Egli H.R. und Fehn K. (eds.). Siedlungsprozesse an der Höhengrenze der Ökumene. Am Beispiel der Alpen. Verlag Siedlungsforschung, Bern/Bonn.

Ammann B. and Wick L. 1993. Analysis of fossil stomata of conifers as indicators of the alpine tree line fluctuations during the Holocene. In: Frenzel B. (ed.). Oscillations of the Alpine and Polar Tree Limits in the Holocene. Paläoklimaforschung. Fischer, Stuttgart, 175-185.

Barnekow L. and Sandgren P. 2001. Palaeoclimate and tree-line changes during the Holocene based on pollen and plant macrofossil records from six lakes at different altitudes in northern Sweden. Rev. Palaeobot. Palynol. 117: 109-118.

Baudais D., Curdy P., David-Elbiali M. et May O. 1990. La néolithisation du Valais: Modèles de peuplement et premier bilan de la prospection archéologique du Valais (Suisse). In: Biagi P. (ed.). The Neolithisation of the Alpine region. Monogr. di "Natura Bresciana" 13, 159-174

Birks H.H. 2001. Plant macrofossils. In: Smol J.P., Birks H.J.B and Last W.M. (eds.). Tracking Environmental Change Using Lake Sediments, Vol. 3. Kluwer, Dordrecht, 49-74.

Birks H.J.B. and Birks H.H. 1980. Quaternary Palaeoecology. Edward Arnold, London.

Bortenschlager S. 1972. Der pollenanalytische Nachweis von Gletscher- und Klimaschwankungen in Mooren der Ostalpen. Ber. Dtsch. bot. Ges. 85: 113-122.

Bradley R.S. 1999. Paleoclimatology-Reconstructing climates of the Quarternary. Academic Press, San Diego.

Burga C.A. 1991. Vegetation History and Paleoclimatology of the Middle Holocene - Pollen Analysis of Alpine Peat Bog Sediments, Covered Formerly by the Rutor Glacier, $2510 \mathrm{M}$ (Aosta Valley, Italy). Glob. Ecol. Biogeogr. Lett. 1: 143-150.

Carrara P.E., Trimble D.A. and Rubin M.1991. Holocene treeline fluctuations in the northern San Juan Mountains, Colorado, USA, as indicated by radiocarbon-dated conifer wood. Arct. Alp. Res. 23: 233-246.

Chaix L. 1990. La faune de Vex-Le-Château (Valais, Suisse; du Néolithique moyen au Bronze final). ASSPA 73: 47-50.

Curdy P., Leuzinger-Piccand C. und Leuzinger U. 1998. Ein Felsabri auf 2'600 m ü.M. am Fusse des Matterhorns - Jäger, Händler und Hirten im Hochgebirge. Archäol. Schweiz 21/2: 65-71.

Ellenberg H. 1996. Vegetation Mitteleuropas mit den Alpen in ökologischer Sicht, 5. Aufl. Ulmer, Stuttgart.

Godet J.-D. 1998. Einheimische Bäume und Sträucher. 5. Aufl., 2001. Arboris, HinterkappelenBern.

Grimm E.C. 1992. Tilia version 1.11 and Tilia*graph version 1.18. Illlinois State Museum, Research and Collections Center, Springfield.

Haas J.N., Richoz I., Tinner W. and Wick L. 1998. Synchronous Holocene climatic oscillations recorded on the Swiss Plateau and at timberline in the Alps. Holocene 8: 301-309.

Katz N.J., Katz S.V. and Kipiani M.G. 1965. Atlas and keys of fruits and seeds occurring in the Quaternary deposits of the USSR. NAUKA, Moskow.

Kullman L. 1995. Holocene tree-limit and climate history from the Scandes Mountains, Sweden. Ecology 76: 2490-2502.

Kupfer J.A. and Cairns D.M. 1996. The suitability of montane ecotones as indicators of global climatic change. Prog. Phys. Geogr. 20: 273-291.

Lang G. 1994. Quartäre Vegetationsgeschichte Europas. Methoden und Ergebnisse. Fischer, Jena. 
Lang G. and Tobolski K. 1985. Hobschensee - Late-Glacial and Holocene environment of a lake near the timberline. Diss. Bot. 87: 209-228.

Markgraf V. 1969. Moorkundliche und vegetationsgeschichtliche Untersuchungen an einem Moorsee an der Waldgrenze im Wallis. Bot. Jahrb. 89: 1-69.

Merkt J. und Streif H.J. 1970. Stechrohr-Bohrgerät für limnische und marine Lockersedimente. Geol. Jahrb. 88: 137-148.

Reasoner M.A. and Hickman M. 1989. Late Quaternary environmental change in the Lake O'Hara region, Yoho National Park, British Columbia. Palaeogeogr. Palaeoclimatol. Palaeoecol. 72: 291-316.

Reasoner M.A., Davies P.T. and Osborn G. 2001. Evaluation of proposed early-Holocene advances of alpine glaciers in the North Cascade Range, Washington State, USA: constraints provided by palaeoenvironmental reconstructions. Holocene 11: 607-611.

Schneider R. and Tobolski K. 1985. Lago di Ganna - Late-glacial and Holocene environments of a lake in the Southern Alps. Diss. Bot. 87: 229-271.

Schoch W.H., Pawlik B. und Schweingruber F.H. 1988. Botanische Makroreste: ein Atlas zur Bestimmung häufig gefundener und ökologisch wichtiger Pflanzensamen. Haupt, Bern.

Schweingruber F.H. 1990. Mikroskopische Holzanatomie: Formenspektren mitteleuropäischer Stamm- und Zweighölzer zur Bestimmung von rezentem und subfossilem Material. WSL, Birmensdorf.

Stöckli W.E. 1995. Geschichte des Neolithikums in der Schweiz. In: Stöckli W.E., Niffeler U. und Gross-Klee E. (Hrsg.). Die Schweiz vom Paläolithikum bis zum frühen Mittelalter Neolithikum. Verlag Schweizerische Gesellschaft für Ur- und Frühgeschichte, Basel, 19-52.

Stuiver M., Reimer P.J., Bard E., Beck J.W., Burr G.S., Hughen K.A., Kromer B., McCormac G., van der Pflicht J and Spurk M. 1998. INTCAL 98 radiocarbon age calibration, 24,000 - 0 cal BP. Radiocarbon 40: 1041-1083.

Tinner W. 1993. Holozäne Veränderungen der oberen Waldgrenze. Diplomarbeit der Universität Bern.

Tinner W. 1994. Quartärbotanische Untersuchungen zur Vegetationsgeschichte der Alpe d`Essertse (Hérémence, Wallis). Bull. Murithienne 112: 79-96.

Tinner W. and Ammann B. 2001. Timberline paleoecology in the Alps. PAGES News, 9, 9-11.

Tinner W., Ammann B. and Germann P. 1996. Treeline fluctuations recorded for 12,500 years by soil profiles, pollen, and plant macrofossils in the central Swiss Alps. Arct. Antarc. Alp. Res. 28: 131-147.

Tinner W. and Kaltenrieder P. 2005. High-resolution macrofossil analyses reveal rapid responses of high-mountain vegetation to early-Holocene environmental changes in the Alps. J. Ecol., in press.

Tinner W. and Theurillat J.P. 2003. Uppermost limit, extent, and fluctuations of the timberline and treeline ecocline in the Swiss Central Alps during the past 11,500 years. Arct. Antarct. Alp. Res. 35: 158-169.

Tobolski K. and Ammann B. 2000. Macrofossils as records of plant responses to rapid Late Glacial climatic changes at three sites in the Swiss Alps. Palaeogeogr. Palaeoclimatol. Palaeoecol. 159: 251-259.

Tranquillini W. 1979. Physiological ecology of the Alpine timberline. Springer, Berlin.

Vallet J.-M. 1950. Etude géologique et pétrographique de la partie inférieure du Val d'Hérens et du Val d'Hérémence (Valais). Leemann, Zürich.

Wegmüller S. und Lotter A.F. 1990. Palynostratigraphische Untersuchungen zur spät- und postglazialen Vegetationsgeschichte der nordwestlichen Kalkvoralpen. Bot. Helv. 100: 37-73.

Welten M. 1982. Vegetationsgeschichtliche Untersuchungen in den westlichen Schweizer Alpen: Bern-Wallis. Denkschr. Schweiz. Nat.forsch. Ges. 95: 1-104.

Wick L. 1994. Early-Holocene reforestation and vegetation change at a lake near the Alpine forest limit: Lago Basso (2'250 m a.s.1.), Northern Italy. Diss. Bot. 234: 555-563.

Wick L. and Tinner W. 1997. Vegetation changes and timberline fluctuations in the Central Alps as indicator of Holocene climatic oscillations. Arct. Alp. Res. 29: 445-458.

Zoller H. 1977. Alter und Ausmass postglazialer Klimaschwankungen in den Schweizer Alpen. In: Frenzel B. (Hrsg.) Dendrochronologie und postglaziale Klimaschwankungen in Europa. Steiner, Wiesbaden, 171-281. 